\title{
Autonomous Wary Collision Avoidance
}

Victor Fors, Björn Olofsson and Lars Nielsen

The self-archived postprint version of this journal article is available at Linköping University Institutional Repository (DiVA):

http://urn.kb.se/resolve?urn=urn:nbn:se:liu:diva-170507

N.B.: When citing this work, cite the original publication.

Fors, V., Olofsson, B., Nielsen, L., (2020), Autonomous Wary Collision Avoidance, IEEE Transactions on Intelligent Vehicles. https://doi.org/10.1109/TIV.2020.3029853

Original publication available at:

https://doi.org/10.1109/TIV.2020.3029853

Copyright: Institute of Electrical and Electronics Engineers

http://www.ieee.org/index.html 


\title{
Autonomous Wary Collision Avoidance
}

\author{
Victor Fors, Björn Olofsson, and Lars Nielsen
}

\begin{abstract}
Handling of critical situations is an important part in the architecture of an autonomous vehicle. A controller for autonomous collision avoidance is developed based on a wary strategy that assumes the least tire-road friction for which the maneuver is still feasible. Should the friction be greater, the controller makes use of this and performs better. The controller uses an acceleration-vector reference obtained from optimal control of a friction-limited particle, whose applicability is verified by using numerical optimization on a full vehicle model. By employing an analytical tire model of the tire-road friction limit, to determine slip references for steering and body-slip control, the result is a controller where the computation of its output is explicit and independent of the actual tire-road friction. When evaluated in real-time on a high-fidelity simulation model, the developed controller performs close to that achieved by offline numerical optimization.
\end{abstract}

Index Terms-Autonomous vehicles, obstacle avoidance, control design, optimal control, vehicle dynamics, vehicle safety.

\section{INTRODUCTION}

A UTONOMY of vehicles is an area with significant ongoing development and also with high expectations from the general public. Accidents involving vehicles using higher levels of autonomy receive significant attention in media, and the acceptance of self-driving vehicles therefore appears to be tied to their capability to avoid accidents. When a pedestrian steps out in front of a self-driving vehicle, how should the vehicle react? If the vehicle can stop in time, the intelligent action should be to brake, but is it certain that the road friction is high enough for the vehicle to stop before reaching the pedestrian? Collision may be unavoidable, but the intelligent behavior could be to be wary, and act according to the worst case for which collision is still avoidable. This research considers a scenario where the vehicle is to autonomously avoid a single obstacle by front-wheel steering and individual four-wheel braking. It is natural that in such critical situations the dynamics of vehicle and tires are important to account for.

One approach to develop a controller for collision avoidance is to separate the problem into path planning and path following as in [1]-[3]. With the advances in computational power, Model Predictive Control (MPC) has gained popularity, which was the method used for trajectory following in [1], [2], [4]. MPC can also be used for simultaneous motion planning and control,

Manuscript received XXX, XX, 2020; revised XXX, XX, 2020. The authors are members of the ELLIIT Strategic Area for ICT research, supported by the Swedish Government. This work was partially supported by the Wallenberg AI, Autonomous Systems and Software Program (WASP) funded by the Knut and Alice Wallenberg Foundation. (Corresponding author: Victor Fors.)

Victor Fors, Björn Olofsson, and Lars Nielsen are with the Division of Vehicular Systems, Department of Electrical Engineering, Linköping University, SE 58183 Linköping, Sweden (email: victor.fors@liu.se; bjorn.olofsson@liu.se; lars.nielsen@liu.se).

Björn Olofsson is also with the Department of Automatic Control, Lund University, SE-22100 Lund, Sweden (email: bjorn.olofsson@ control.lth.se). which has been applied to the problem of collision avoidance in [5]-[10], and the related problem of lane change in [11]. The predictive nature of MPC allows for complex planning while considering the dynamics of the vehicle, but when applied to the nonlinear models necessary to capture vehicle behavior up to the limits of friction, the computational cost to find the optimal trajectory has to be handled and often results in a nonconvex problem where convergence is not guaranteed. These methods use friction as a parameter that needs to be deduced or estimated in some way. Friction can be difficult to estimate before entering a critical maneuver and can change from one road segment to the next.

Aiming for a method independent of friction, computationally cheap, and applicable to emergency collision avoidance where driving at-the-limit of friction is desired, another direction of research is used here, namely to directly consider the forces or acceleration acting on the vehicle. This is natural when considering maneuvers where the vehicle operates at-the-limit of friction. As demonstrated in [12] in a post-impact scenario, the problem of control allocation can in such situations be simplified by determining an acceleration reference rather than explicitly following a path or trajectory. This concept is also used in the Modified Hamiltonian Algorithm (MHA) [13], where the trade-off between yaw-moment control and centerof-mass acceleration is determined by an iterative process, rather than through an optimization process as in [12]. The MHA controller has been applied to the problems of collision avoidance and minimum-time lane change in [14], [15]. In [16], a steering controller for lane-departure prevention was developed that exploits how optimal maneuvers tend to push the individual tires close to their friction limit, allowing explicit computation of the desired tire-slip angles from an acceleration reference. This was extended in [17] by using an empirical tire model that only describes the tire at the friction limit, and incorporating yaw-moment control using the MHA method while taking advantage of the computed desired tire-slip angles. The controller in this paper is developed further based on the ideas in [12]-[17].

The main contribution of this paper is the controller that is independent of actual friction and is fully explicit as outlined in Section II and described in detail in Section V. One key contribution is the wary principle as described in Section II. The analytical solutions obtained from optimal control of a friction-limited particle model [6] are used, whose applicability are verified in Section III by using numerical optimization on a full vehicle model. Another key contribution is that instead of using an empirical tire model as in [17], a simple analytical model of the slip angle at the tire-road friction limit is derived (see Section IV). The controller is demonstrated on a highfidelity simulation model in CarMaker [18] to be capable of collision avoidance up to the limit of friction (see Section VI). 


\section{OVERVIEW OF CONTROLlER PRINCIPLES}

For straight-line braking there are systems, such as the anti-lock braking system (ABS) (see, e.g., [19]), that do not require prior information about the road friction to operate, but what about passing maneuvers? As detailed in Section V, this paper develops such a passing controller independent of actual tire-road friction. In a first step for avoidance a wary principle is used, which means that the avoidance problem is solved for the least possible friction for which it is still possible to avoid the obstacle. Initially, when an obstacle needs to be handled like in the scenario in Fig. 1, a first decision is whether to brake in order to stop before reaching the obstacle or to initiate a passing maneuver. This is treated in Section III, where the decision of whether to stop or pass is independent of the friction, and is determined solely by the obstacle-related angle $\gamma$. If the decision is to perform a passing maneuver, a reference for the necessary center-of-mass motion to pass the obstacle is determined and updated as the maneuver progresses. From the obtained motion, the path is not used since it would mean passing unnecessarily close to the obstacle even when the friction is better than the least possible. Instead of using the path, the controller uses the obtained acceleration vector as a reference in a first of several steps of refinement. The design then continues by, so to say, cascading the center-of-mass acceleration vector to desired acceleration vectors at each wheel. The wheel-level acceleration vectors are determined to not only control the center-of-mass acceleration, but also to control the yaw acceleration for body-slip control From the wheel-level acceleration references, desired tire-slip angles and braking forces are computed using the nonlinear tire models described in Section IV. Finally, appropriate steering and braking signals are determined. Note that the complete control algorithm described in Section $\mathrm{V}$ is independent of the actual tire-road friction and that it is explicit, consisting of a sequence of assignment statements.

\section{CENTER-OF-MASS ACCELERATION REFERENCE}

When performing maneuvers requiring large accelerations, the resulting motion is largely determined by the constraint on acceleration due to friction. The controller in Section $\mathrm{V}$ is as those in [13]-[17] based on following the acceleration reference retrieved from a friction-limited particle (or point-mass) model. In the friction-limited particle model the maximum acceleration is limited by the friction coefficient $\mu$ and gravitational acceleration constant $g$ :

$$
\ddot{X}=u_{1}, \quad \ddot{Y}=u_{2}, \quad u_{1}^{2}+u_{2}^{2} \leq(\mu g)^{2},
$$

where $X$ and $Y$ are the positional coordinates with corresponding acceleration inputs $u_{1}$ and $u_{2}$. In this section, different strategies are examined for the simple friction-limited particle model and the optimal solution is compared with the results obtained by numerical optimization of a 9-degree-of-freedom (9DoF) vehicle model to verify its applicability. While (1) can be modified with additional constraints, e.g., power-train limits or jerk limits, to increase its validity [15], [20], the solution obtained with the simple circular friction constraint

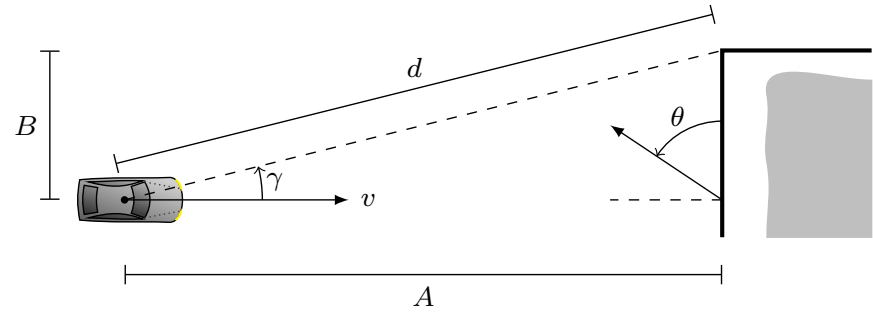

Fig. 1. Collision-avoidance scenario. The vehicle enters from the left with the velocity $v$ and reacts to the obstacle a distance $A$ away from the front of the vehicle. The obstacle region for the vehicle center of mass is marked as thick black lines and spans a distance $B$ in the lateral direction, the angle between the vehicle velocity vector and the corner of the obstacle space is the passing angle $\gamma$, and the distance to the corner of the obstacle space is $d$. The direction of the acceleration reference of the vehicle is marked as the angle $\theta$.

turns out to be similar to that obtained with the 9DoF model (see Section III-B).

When planning vehicle maneuvers there is usually freedom to not only find a feasible path, but also to optimize the trajectory according to a criterion. In the context of critical collision avoidance, examples of such criteria are to perform minimum-time lane change [13], [15], [21], minimum-distance lane change [22], [23], minimum-distance collision avoidance [6], or collision avoidance while minimizing the overshoot in the new lane [14]. Here, considering the wary strategy to be applied by the controller in Section V, the trajectory is planned for the minimum possible friction considering collision avoidance of a single obstacle.

\section{A. Friction-Limited Particle Model}

The scenario examined is the collision-avoidance scenario illustrated by Fig. 1. To handle the car geometry, the obstacle region for the vehicle center of mass is assumed to be an enlarged region of the actual obstacle. The obstacle region takes into account the vehicle length and width, where it can be assumed that the vehicle body slip is kept small by active control. First, a few simple strategies will be examined before examining the optimal solution.

1) Straight-Line Braking: The simplest strategy is to stop before reaching the obstacle. The minimum friction required to stop within the braking distance $A$ when traveling with the velocity $v$ is $\mu=v^{2} /(2 g A)$. When comparing the performance to other maneuvers, it will be shown interesting to compare with the dimensionless, and for straight-line braking invariant quantity

$$
\frac{2 \mu g A}{v^{2}}=1
$$

2) Minimum-Time Lane Change: Applying track-lateral acceleration to avoid the obstacle, corresponding to $\theta=0$ in Fig. 1, is related to the minimum-time lane-change maneuver (see [13], [15], [21]). To avoid the obstacle using this strategy, the vehicle has to travel the distance $B$ in the track-lateral direction before reaching the obstacle. The problem can be stated as (with $t_{f}$ the final time)

$$
X(t)=v t, \quad Y(t)=\mu g t^{2} / 2, \quad X\left(t_{f}\right)=A, \quad Y\left(t_{f}\right)=B .
$$


Solving (3), using $\tan (\gamma)=B / A$, and ordering the left-hand side as (2) gives

$$
\frac{2 \mu g A}{v^{2}}=4 \tan (\gamma)
$$

Examining for which passing angle $\gamma$ the right-hand side of (4) is smaller than 1 (given by straight-line braking) gives that this strategy performs better than straight-line braking up to $\gamma=14^{\circ}$. This does not mean that the obstacle can necessarily be avoided for passing angles below $14^{\circ}$, avoidance is ensured if the right-hand side of (4) is smaller or equal to the actual value of the left-hand side. It can easily be seen that as $\gamma$ approaches zero the required friction coefficient $\mu$ to avoid the obstacle also approaches zero.

3) Constant Curvature Turn: Another simple strategy is the constant curvature turn. The minimum radius of the constant curvature turn is $R=v^{2} /(\mu g)$ and the radius of the required turn to traverse is given by the geometry as the circle equation $A^{2}+(B-R)^{2}=R^{2}$. Solving for the left-hand side expression in (2) gives after simplification

$$
\frac{2 \mu g A}{v^{2}}=\frac{2 A}{R}=2 \sin (2 \gamma)
$$

Computing the value for when the right-hand side is 1 , it is found that the constant curvature turn strategy performs better than straight-line braking up to the passing angle $\gamma=15^{\circ}$.

4) Optimal Avoidance Maneuver: The problem of optimal avoidance of a single obstacle for a friction-limited particle model (1) is studied in detail in [6]. Using the notation of Fig. 1, a derivation is made to give the reader an intuition about the resulting solution. As shown in [6], where the optimal passing maneuver is obtained from the minimization of the longitudinal travel distance, it results in a globally fixed acceleration vector with maximum magnitude. Here, to derive the optimal avoidance maneuver, the optimal constant- $\theta$ passing maneuver is found and compared with straight-line braking.

To find an expression for the constant $\theta$ passing maneuvers that reach the corner of the obstacle for some fixed $\gamma \in(0, \pi / 2)$, the system dynamics (1) is integrated yielding the system

$$
\begin{aligned}
X(t) & =v t-\sin (\theta) \mu g t^{2} / 2, & Y(t) & =\cos (\theta) \mu g t^{2} / 2, \\
X\left(t_{f}\right) & =A=d \cos (\gamma), & Y\left(t_{f}\right) & =B=d \sin (\gamma) .
\end{aligned}
$$

Solving this system gives the expression

$$
\frac{\mu g d}{v^{2}}=\frac{2 \sin (\gamma) \cos (\theta)}{\cos ^{2}(\theta-\gamma)}
$$

or alternatively, as before on the form of (2) as

$$
\frac{2 \mu g A}{v^{2}}=\frac{4 \sin (\gamma) \cos (\gamma) \cos (\theta)}{\cos ^{2}(\theta-\gamma)}
$$

The optimal $\theta$ is at a local minimum, at the limit of $\theta \in[0, \pi / 2)$, or limited by feasibility to not pass through the obstacle. Differentiating (7) to find extrema gives ${ }^{1}$

$$
\begin{aligned}
& \frac{\mathrm{d}\left(\frac{\mu g d}{v^{2}}\right)}{\mathrm{d} \theta}=\frac{2 \sin (\gamma)(2 \cos (\theta) \sin (\theta-\gamma)-\sin (\theta) \cos (\theta-\gamma))}{\cos ^{3}(\theta-\gamma)} \\
&=\frac{\sin (\gamma)(\sin (2 \theta-\gamma)-3 \sin (\gamma))}{\cos ^{3}(\theta-\gamma)} \\
&{ }^{1} 2 \cos (x) \sin (y)-\sin (x) \cos (y)=(\sin (x+y)-3 \sin (x-y)) / 2
\end{aligned}
$$

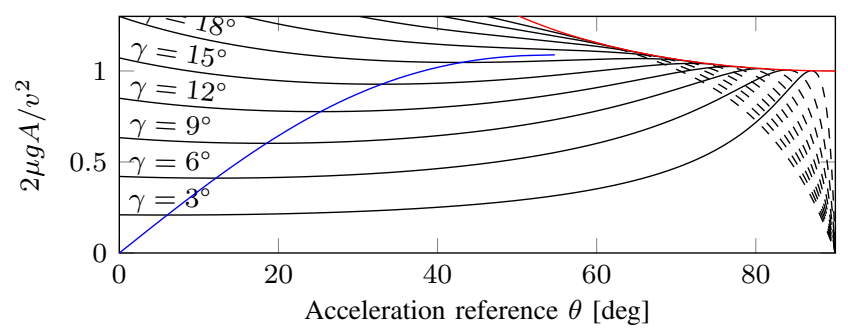

Fig. 2. The black curves show the performance of constant- $\theta$ passing maneuvers given by (8) for different $\gamma$, with values resulting in invalid trajectories that pass through the obstacle as dashed. The blue curve shows the value given by the minimum (10). The red curve shows the value given by the feasibility condition (13).

Because (9) is negative at $\theta=0$ (the minimum-time lanechange maneuver) for $\gamma \in(0, \pi / 2)$, this boundary is not the optimal $\theta$. By setting (9) equal to zero and also analyzing the second derivative it can be shown that if $\gamma<\arcsin (1 / 3) \approx$ $19.5^{\circ}$, there is a local minimum at

$$
\theta=\frac{\gamma+\arcsin (3 \sin (\gamma))}{2} \text {. }
$$

Finally, we examine the upper bound of $\theta$, which at least corresponds to the optimal constant $\theta$ passing maneuver when the minimum in (10) does not exist. A sufficient condition for the trajectories given by (6) to be collision free is to check if the final longitudinal velocity is larger or equal to zero, i.e.,

$$
v-\mu g \sin (\theta) t_{f} \geq 0 \text {. }
$$

Solving for $t_{f}$ in (6) and inserting in (11) gives

$$
\frac{2 \mu g A}{v^{2}} \leq \frac{\cos (\gamma) \cos (\theta)}{\sin (\gamma) \sin ^{2}(\theta)}
$$

Replacing the left-hand side of (12) with (8) and using common trigonometric identities, the condition on $\theta$ is

$$
\theta \leq \arctan \left(\frac{1}{\tan (\gamma)}\right)
$$

The value of (8) as a function of $\theta$ is plotted for different $\gamma$ in Fig. 2, with the line corresponding to the local minimum (10) in blue and the feasibility condition (13) in red. Comparing (8) for the maximum feasible $\theta$ in (13) with the local minimum (10), it is found that the former gives the optimal constant $\theta$ passing maneuver if $\gamma>18.2^{\circ}$. Comparing with the straightline braking case, it is as in [6] found that the passing maneuver can be performed at less friction than straight-line braking when $\gamma<16.7^{\circ}$. The optimal avoidance maneuver for the scenario described by Fig. 1 is thus given by (10) if $\gamma<16.7^{\circ}$, and otherwise by straight-line braking.

5) Performance Comparison: A common way to compare the strategies of straight-line braking and passing is to plot the relation between minimum passing distance and the velocity, with the friction coefficient $\mu$ and obstacle dimension $B$ fixed [5], [21]. In [23], dimensionless variables are used to give a comparison plot that represents the full variation of parameters for minimum-distance lane-change maneuvers. The trade-off between straight-line braking and passing maneuvers is presented in Fig. 3 in a similar way to that in [6], but with 


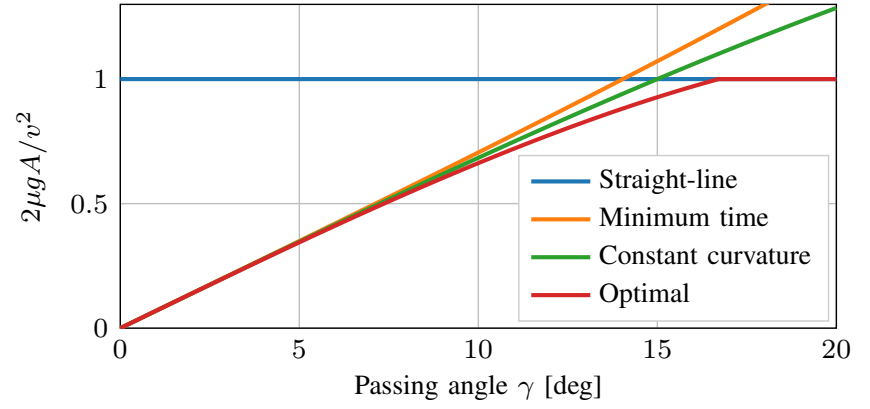

Fig. 3. Friction-limited particle performance for different collision-avoidance maneuvers as a function of the passing angle $\gamma$. Lower is better.

the performance normalized to the performance of straight-line braking. With Fig. 3 it is easy to determine how much better or worse than straight-line braking the other avoidance maneuvers are for different passing angles. For example, at $\gamma=10^{\circ}$, since the ordinate axis is proportional to the minimum friction required, the passing maneuvers can be seen to be able to handle $30 \%$ lower friction than straight-line braking.

\section{B. 9DoF Double-Track Vehicle Model}

To verify that the results from the friction-limited particle model are also applicable for a full vehicle model, numerical optimization is used. For this purpose, the 9DoF double-track vehicle model from [24] designed for numerical optimization is used, which describes the longitudinal, lateral, yaw, pitch, and roll motion of the vehicle chassis, as well as the rotational motion of each wheel. The model includes longitudinal and lateral load transfer and comprises a nonlinear tire model accounting for combined slip from [25]. The parameters of the model are made to be representative to that of the CarMaker model used for simulation. To facilitate optimization, the tire forces are modeled as linear with respect to both vertical load and road friction coefficient $\mu_{\text {road }}$. While simplified, the model is useful in getting a general idea of the optimal behavior and performance limit of a vehicle similar to the high-fidelity model used in simulation.

1) Optimal Control Problem: The optimal control problem is stated as follows:

$$
\begin{aligned}
\operatorname{minimize} & \mu_{\text {road }} \\
\text { subject to } & T_{i, \min } \leq T_{i} \leq 0, i \in\{1,2,3,4\}, \\
& |\delta| \leq \delta_{\max }, \quad|\dot{\delta}| \leq \dot{\delta}_{\max }, \\
& x(0)=x_{0}, \quad g\left(x\left(t_{f}\right)\right) \leq 0, \\
& X \leq A, \quad \dot{X} \geq 0, \\
& \dot{x}=G(x, z, u, p), \quad h(x, z, u, p)=0 .
\end{aligned}
$$

The functions $G$ and $h$ define the vehicle dynamics as a semiexplicit differential algebraic equation system with the dynamic state vector $x$, algebraic variable vector $z$, input vector $u$, and parameter vector $p$. The dynamic state vector $x$ consists of the 19 states that are differentiated in the vehicle model, while the algebraic variable vector $z$ consists of the variables not differentiated. The inputs to the model in $u$ are the steering rate of the front axle $\dot{\delta}$, and the braking torque $T_{i}$ at each wheel. The parameter vector $p$ consists of the vehicle and road parameters, including the road friction parameter $\mu_{\text {road }}$. The constraints on the steering angle $\delta$ and steering rate $\dot{\delta}$ are $\delta_{\max }=0.5 \mathrm{rad}$ and $\dot{\delta}_{\text {max }}=2 \mathrm{rad} / \mathrm{s}$, respectively. With subscript $i$ denoting the $i$ th wheel of the vehicle, the braking torque $T_{i}$ is constrained by the maximum tire-road braking force as $T_{i, \min }=-\mu_{x, i} R_{e} F_{z, i}$, where $\mu_{x, i}$ is the longitudinal tire-road friction coefficient, $R_{e}$ is the effective wheel radius, and $F_{z, i}$ is the vertical tire load. The initial condition is determined by $x_{0}$ and the terminal inequality constraints by the function $g$.

Because the collision-avoidance problem is nonconvex, different functions $g$ are used to guide the solver to the two different strategies of stopping before reaching the obstacle and passing it. To examine the straight-line braking maneuver, the terminal inequality constraint is set to $g\left(x\left(t_{f}\right)\right)=\dot{X}\left(t_{f}\right)-10^{-9}$, where the small subtracted number accounts for the fact that the vehicle model is not well defined at zero velocity. To examine the passing maneuver, the terminal inequality constraint is set to $g\left(x\left(t_{f}\right)\right)=B-Y\left(t_{f}\right)$. The optimal solution is then taken as the strategy requiring the smallest friction. The optimizations are carried out for the distance $A=20 \mathrm{~m}$, the initial velocity $v=70 \mathrm{~km} / \mathrm{h}$, and varying passing angles $\gamma$. The optimal control problem (14) is solved numerically by direct collocation [26] using the JModelica.org platform [27], where necessary Jacobians and Hessians are computed using algorithmic differentiation by the CasADi tool [28]. The resulting nonlinear optimization program is solved using the nonlinear solver IPOPT [29] with the linear solver MA57 [30]

2) Resulting Forces and Moment: The optimizations do not directly contain an acceleration reference $\theta$, but using the procedure in [31], the corresponding reference can be found for a single-obstacle at-the-limit avoidance maneuver such as the one examined. The control force component $F_{c, y}$ turns out to be of particular interest. It is defined as the globally fixed force component perpendicular to the final velocity vector and is the force that stops the approach of the vehicle towards the obstacle [31]. Solving (14) for the passing maneuver, the force component $F_{c, y}$ is then computed using the vehicle longitudinal and lateral forces, $F_{x}$ and $F_{y}$, by

$$
F_{c, y}=-\sin \left(\psi_{v}\left(t_{f}\right)-\psi\right) F_{x}+\cos \left(\psi_{v}\left(t_{f}\right)-\psi\right) F_{y},
$$

where the angle $\psi$ is the vehicle orientation and $\psi_{v}\left(t_{f}\right)$ defines the orientation of the final velocity vector. Similar to how $\theta$ is constant for the optimal single-obstacle avoidance maneuver for a friction-limited particle model [6], the optimal input $u^{*}$ for a simplified vehicle model can be shown to satisfy the necessary condition [31]

$$
u^{*}=\underset{u \in U}{\arg \max }\left(F_{c, y}(u)+\lambda M_{z}(u)\right),
$$

where the Lagrange multiplier $\lambda$ determines the trade-off between yaw-moment $M_{z}$ control and maximizing $F_{c, y}$, and $U$ is the set of allowed inputs. It is thus expected that the forces will be on the boundary of the attainable control force $F_{c, y}$ and the yaw moment $M_{z}$. The attainable forces arising from different degrees of individual braking from all four wheels can as in [32] be approximated by assuming that the longitudinal tire states can instantaneously change, which influence both 




Fig. 4. Attainable force volume from braking during the optimal avoidance maneuver obtained for $\gamma=10^{\circ}$, which corresponds to the obstacle dimension $B \approx 3.5 \mathrm{~m}$. The optimal trajectory is shown as a solid line. The trajectory corresponding to maximum attainable $F_{c, y}$ is shown as a dashed line. Projections are cast on the $F_{c, y}$ and $M_{z}$ axes to make it more visible how close the optimal trajectory is to the respective attainable limit.

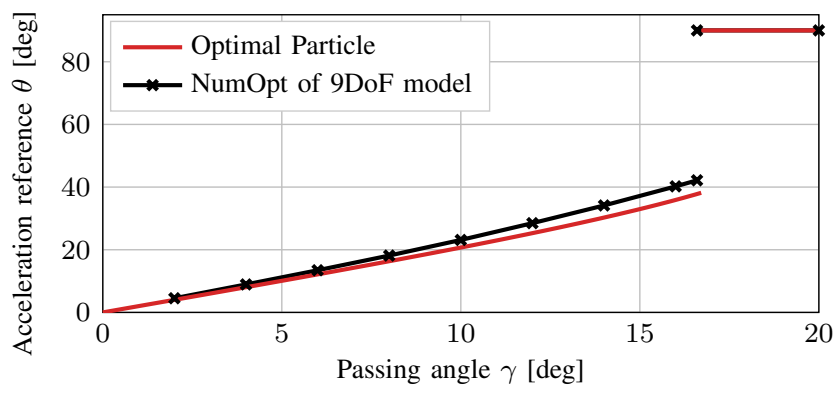

Fig. 5. Comparison of optimal particle reference with the equivalent given by numerical optimization.

longitudinal and lateral tire forces. The attainable forces during a maneuver were illustrated in [12] by creating plots of them at different time instants. Here, to visualize the attainable forces during one maneuver obtained by solving (14), Fig. 4 shows the attainable force volume introduced in [31], where it is seen that the force is indeed on the boundary of $F_{c, y}$ and $M_{z}$ as indicated by (16). As also observed in [31] for a similar maneuver, it can be seen that there is not much active control of the yaw moment, with the trajectory being close to maximum $F_{c, y}$. For the optimal particle, the direction of $F_{c, y}$ is given by $\theta$. Defining the acceleration reference $\theta$ as the direction of $F_{c, y}$ for the $9 \mathrm{DoF}$ model when performing a passing maneuver, i.e., $\theta=\psi_{v}\left(t_{f}\right)$, and as $90^{\circ}$ when performing a stopping maneuver (see Fig. 1), the angle $\theta$ obtained from numerical optimization (NumOpt) of the 9DoF model is plotted in Fig. 5. When compared with $\theta$ obtained by analytical optimal control of the particle model, the resulting references can be seen to be a good match. The observation that the acceleration reference $\theta$ given by numerical optimization is a bit larger is expected, given that the additional degrees of freedom and actuator limitations mean that the vehicle can not perform as well as obtained with the particle representation and therefore has to compensate. This difference in performance can be seen in Fig. 11 for different passing angles $\gamma$.

\section{Tire Modeling FOR REAL-Time CONTROL}

It is clear from Section III that it is interesting to maximize the vehicle forces according to an acceleration reference $\theta$. This section presents tire modeling suitable for at-the-limit control and derives an analytical expression for how to orient the wheels to maximize the tire forces given an acceleration reference.

\section{A. Lateral Tire Forces}

A common quantity when modeling lateral tire forces is the slip angle $\alpha_{i}$. It describes the angle at which the tire moves as

$$
\tan \left(\alpha_{i}\right)=-v_{y, i} / v_{x, i},
$$

where $\left(v_{x, i}, v_{y, i}\right)$ is the tire velocity vector. For small tire slips, the lateral tire forces $F_{y, i}$ are linear with respect to $\alpha_{i}$. The slope at this linear region is called the cornering stiffness $C_{\alpha, i}$ and is defined as [33]

$$
C_{\alpha, i}=\left.\frac{\partial F_{y, i}}{\partial \alpha_{i}}\right|_{\alpha_{i}=0} .
$$

It is beneficial to have few parameters to estimate. A model of the nominal lateral tire force $F_{y 0, i}$ (nominal referring to the absence of a longitudinal tire force $F_{x, i}$ ) dependent only on the lateral tire-road friction coefficient $\mu_{y, i}$, cornering stiffness $C_{\alpha, i}$, and vertical tire load $F_{z, i}$ is the Fiala brush model [25]:

$$
F_{y 0, i}=\left\{\begin{array}{cc}
C_{\alpha, i} \tan \left(\alpha_{i}\right) & \\
-\frac{C_{\alpha, i}^{2}\left|\tan \left(\alpha_{i}\right)\right| \tan \left(\alpha_{i}\right)}{3 \mu_{y, i} F_{z, i}} & \\
+\frac{C_{\alpha, i}^{3} \tan ^{3}\left(\alpha_{i}\right)}{27 \mu_{y, i}^{2} F_{z, i}^{2}}, & \text { if }\left|\alpha_{i}\right|<\alpha_{\mathrm{sl}}, \\
\mu_{y, i} F_{z, i} \operatorname{sign}\left(\alpha_{i}\right), & \text { otherwise, }
\end{array}\right.
$$

where $\alpha_{\mathrm{sl}}$ is the slip angle where total sliding occurs, which is computed as

$$
\alpha_{\mathrm{sl}}=\arctan \left(3 \mu_{y, i} F_{z, i} / C_{\alpha, i}\right) .
$$

A comparison between the Fiala brush model and the CarMaker tire model (map-based model described by measurement data) is seen in Fig. 6, where the normalized cornering stiffness $\hat{C}_{\alpha}=C_{\alpha, i} / F_{z, i}$ is approximated to $18 \mathrm{rad}^{-1}$, and the vertical force and friction coefficients are adopted from the CarMaker data. The lateral tire-road friction coefficient $\mu_{y, i}$ decreases with increased vertical load from 1.11 at $F_{z, i}=2 \mathrm{kN}$ to 0.93 at $F_{z, i}=6 \mathrm{kN}$. The corresponding values for the longitudinal coefficient $\mu_{x, i}$ is 1.11 and 0.95 , respectively. At nominal tire load (achieved at zero longitudinal and lateral acceleration), the compound longitudinal and lateral friction coefficients of the car resulting from the individual tires are $\mu_{x}=1.07$ and $\mu_{y}=1.06$, respectively.

\section{B. Combined Steering and Braking}

The controller in Section $\mathrm{V}$ performs simultaneous steering and braking, and there is thus a need to model the influence of combined longitudinal and lateral tire forces. It is common to consider a friction constraint for individual tires as

$$
\left(F_{x, i} / \mu_{x, i}\right)^{2}+\left(F_{y, i} / \mu_{y, i}\right)^{2} \leq F_{z, i}^{2}
$$




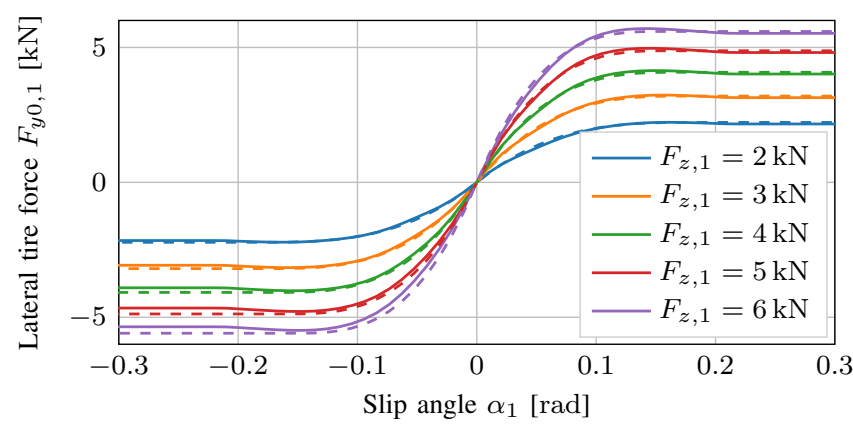

Fig. 6. Lateral tire force of the front-left tire as function of the slip angle for different vertical tire loads. The CarMaker model is shown as solid curves and the Fiala brush model (19) fit in dashed.
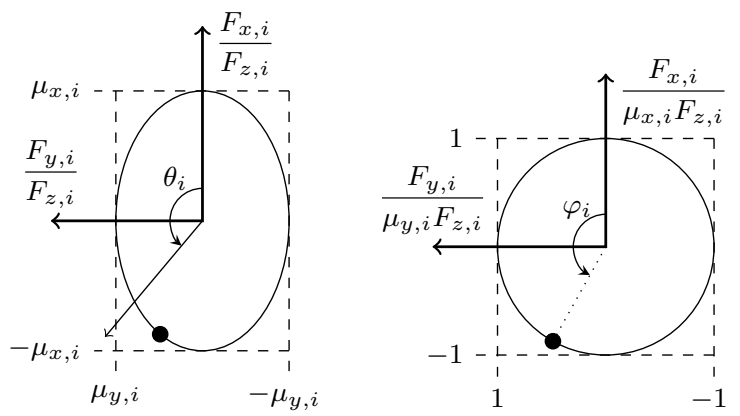

Fig. 7. Friction ellipse describing the tire-road friction limit. The left figure shows the maximum force in the direction indicated by the angle $\theta_{i}$ as a filled circle. The right figure shows how for the normalized ellipse the same force along the boundary is described by the angle $\varphi_{i}$.

This is commonly referred to as the friction circle [25] or friction ellipse [33] and the constraint is illustrated in Fig. 7. To describe the unsaturated behavior based on this condition, the model later used to compute appropriate braking signals in Section V-B2 is the friction-ellipse based model [33]

$$
F_{y, i}=F_{y 0, i} \sqrt{1-\left(\frac{F_{x, i}}{\mu_{x, i} F_{z, i}}\right)^{2}} .
$$

For driving at-the-limit of friction, this model is, however, not suitable to compute appropriate slip angles. To reach the tirefriction limit (21) according to (22), the slip angle needs to be such that the maximum $F_{y 0, i}$ is achieved, since for all other $F_{y 0, i}$ the friction limit can only be reached at $F_{x, i}= \pm \mu_{x, i} F_{z, i}$. This is not in accordance with real tire data [34], and would in a control system lead to a discontinuous jump of the desired slip angle when the desired tire force moves between the left and the right half of the friction ellipse.

\section{Analytical Friction-Limit Tire Model}

In [17], the tire forces at-the-limit of friction were modeled by reducing a full tire model to one that only models the tire force at the friction limit and fitting a simplified version of Pacejka's Magic Formula [25] to it. Here, an analytical approach is used instead to approximate which slip angle $\alpha_{i}$ that corresponds to which part of the friction limit, which is based on the same assumptions as the full tire model in [34].
For combined tire slips close to total sliding, the tire forces normalized with respect to the peak friction at total sliding are co-linear to the normalized tire-slip vector $\left(\hat{\sigma}_{x, i}, \hat{\sigma}_{y, i}\right)$ [34]. These are defined as [35]

$$
\hat{\sigma}_{x, i}=\frac{v_{x, i}-\omega_{i} R_{e}}{\sigma_{x, \mathrm{sl}} \omega_{i} R_{e}}, \quad \hat{\sigma}_{y, i}=\frac{v_{y, i}}{\sigma_{y, \mathrm{sl}} \omega_{i} R_{e}},
$$

where $\omega_{i}$ is the wheel's rotational speed, and $\sigma_{x, \mathrm{sl}}, \sigma_{y, \mathrm{sl}}$ are the normalizing slips where total sliding occurs. The resulting tire forces are

$$
F_{x, i}=-\frac{\hat{\sigma}_{x, i}}{\hat{\sigma}_{i}} F_{x 0, i}\left(\hat{\sigma}_{i}\right), \quad F_{y, i}=-\frac{\hat{\sigma}_{y, i}}{\hat{\sigma}_{i}} F_{y 0, i}\left(\hat{\sigma}_{i}\right),
$$

where $\hat{\sigma}_{i}=\sqrt{\hat{\sigma}_{x, i}^{2}+\hat{\sigma}_{y, i}^{2}}$. At the total sliding condition at the friction limit, it holds that $\hat{\sigma}_{i}=1, F_{x 0, i}=\mu_{x, i} F_{z, i}$, and $F_{y 0, i}=\mu_{y, i} F_{z, i}$. Combining this with the forces described by the friction ellipse (Fig. 7), the angle $\varphi_{i}$ is introduced to describe the position along the friction-ellipse boundary and it is related to the tire-slip vector by

$$
\begin{aligned}
& F_{x, i}=\mu_{x, i} F_{z, i} \cos \left(\varphi_{i}\right)=-\mu_{x, i} F_{z, i} \hat{\sigma}_{x, i}, \\
& F_{y, i}=\mu_{y, i} F_{z, i} \sin \left(\varphi_{i}\right)=-\mu_{y, i} F_{z, i} \hat{\sigma}_{y, i} .
\end{aligned}
$$

The slip quantity $\hat{\sigma}_{y, i}$ is not convenient to use in a feedback steering controller since it is highly dependent on the wheel speed $\omega_{i}$. Rewriting $\hat{\sigma}_{y, i}$ in terms of the slip angle $\alpha_{i}$ gives

$$
\hat{\sigma}_{y, i}=-\tan \left(\alpha_{i}\right) \frac{v_{x, i}}{\sigma_{y, \mathrm{sl}} \omega_{i} R_{e}} .
$$

The ratio $v_{x, i} /\left(\omega_{i} R_{e}\right)$ is calculated from (23) and (25a) to

$$
\frac{v_{x, i}}{\omega_{i} R_{e}}=1-\sigma_{x, \mathrm{sl}} \cos \left(\varphi_{i}\right)
$$

An expression for the desired slip angle $\alpha_{i}^{*}$ for a given $\varphi_{i}$ can now be obtained from (25b), (26), and (27) as

$$
\tan \left(\alpha_{i}^{*}\right)=\frac{\omega_{i} R_{e}}{v_{x, i}} \sigma_{y, \mathrm{sl}} \sin \left(\varphi_{i}\right)=\frac{\sigma_{y, \mathrm{sl}} \sin \left(\varphi_{i}\right)}{1-\sigma_{x, \mathrm{sl}} \cos \left(\varphi_{i}\right)} .
$$

It can be noted that the peak longitudinal force typically occurs long before wheel lock, $\omega_{i}=0$. Thus, the ratio $\omega_{i} R_{e} / v_{x, i}$ is approximated to 1 . Further, noting that total sliding in terms of $\alpha_{i}$ occurs at $\alpha_{\mathrm{sl}}$ and using a small-angle approximation for $\alpha_{i}$, the desired slip angle is approximated to the simple expression

$$
\alpha_{i}^{*}=\alpha_{\mathrm{sl}} \sin \left(\varphi_{i}\right) .
$$

In the controller in Section $\mathrm{V}$, the maximum force achievable in the direction defined by $\theta_{i}$ is sought (see Fig. 7). The relation between $\varphi_{i}$ and $\theta_{i}$ is in [16] found by solving it as an optimization problem giving:

$$
\varphi_{i}=\arctan 2\left(\mu_{y, i} \sin \left(\theta_{i}\right), \mu_{x, i} \cos \left(\theta_{i}\right)\right) .
$$

\section{Online AVoidance Control}

With reference to the overview in Fig. 8, this section presents an explicit controller that performs autonomous collision avoidance by steering and braking without using any estimation of the actual road friction. The vehicle parameters used in the controller are summarized in Table I and the vehicle variables that are assumed to be available from measurement or estimation are summarized in Table II. 


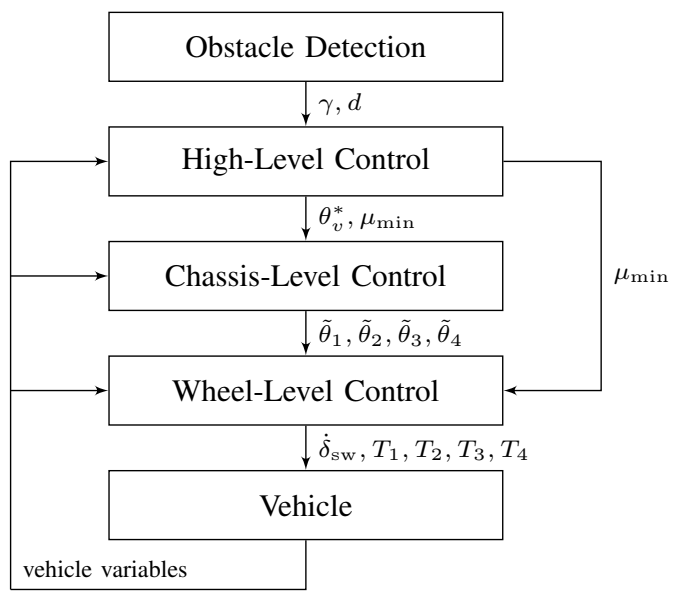

Fig. 8. Control layout. The vehicle variables are defined in Table II.

TABLE I

VEHICLE PARAMETERS USED IN THE CONTROLLER

\begin{tabular}{lccc}
\hline Description & Notation & Value & Unit \\
\hline Distance to front axle & $l_{f}$ & 1.043 & $\mathrm{~m}$ \\
Distance to rear axle & $l_{r}$ & 1.637 & $\mathrm{~m}$ \\
Track width & $w$ & 1.510 & $\mathrm{~m}$ \\
Yaw moment of inertia & $I_{z z}$ & 1730 & $\mathrm{~kg} \mathrm{~m}^{2}$ \\
Effective wheel radius & $R_{e}$ & 0.293 & $\mathrm{~m}$ \\
Steering ratio & $k_{\delta}$ & 19.8 & - \\
Normalized cornering stiffness & $\hat{C}_{\alpha}$ & 18 & $\mathrm{rad}^{-1}$ \\
Tire friction ratio & $\mu_{x, i} / \mu_{y, i}$ & 1 & - \\
\hline
\end{tabular}

TABLE II

ROAD AND VEHICLE VARIABLES USED IN THE CONTROLLER

\begin{tabular}{llc}
\hline Description & Notation & Unit \\
\hline Passing angle & $\gamma$ & $\mathrm{rad}$ \\
Avoidance distance & $d$ & $\mathrm{~m}$ \\
Velocity & $v_{x}, v_{y}$ & $\mathrm{~m} / \mathrm{s}$ \\
Acceleration & $a_{x}, a_{y}$ & $\mathrm{~m} / \mathrm{s}^{2}$ \\
Yaw rate & $\dot{\psi}$ & $\mathrm{rad} / \mathrm{s}$ \\
Yaw acceleration & $\ddot{\psi}$ & $\mathrm{rad} / \mathrm{s}^{2}$ \\
Front steering angles & $\delta_{1}, \delta_{2}$ & $\mathrm{rad}$ \\
Vertical tire loads & $F_{z, 1}, F_{z, 2}, F_{z, 3}, F_{z, 4}$ & $\mathrm{~N} \mathrm{~m}$ \\
\hline
\end{tabular}

With reference to the scenario in Fig. 1, the first step is to update $\gamma$ and $d$ (from sensors), and the avoidance (to the left) is successful once $\gamma \leq 0$. The next major step is to apply the wary approach, i.e., to assume maximum slipperiness possible to handle. The High-Level Control step computes the minimum friction coefficient $\mu_{\min }$ for which avoidance is feasible and the corresponding center-of-mass acceleration reference $\theta_{v}^{*}=\theta^{*}$ $\psi$ (see Fig. 9), expressed relative to the vehicle orientation $\psi$, which results in successful avoidance. These are approximated by using the solution to optimal collision avoidance for a friction-limited particle (see Section III-A4). Using the control principle of aiming for the constant acceleration reference $\theta^{*}$ as obtained for the particle model was justified for a 9DoF model in Section III-B (see in particular Fig. 5). Summing up this step, rather than trying to estimate the actual tire-road friction, the variables $\theta_{v}^{*}$ and $\mu_{\min }$ are passed on.

Now, a vehicle is not a particle so on a chassis level the yaw dynamics needs to be handled. To do this, the ChassisLevel Control (see Section V-A) takes further advantage of the approximate references from the particle model to compute the next set of reference values for the Wheel-Level Control. First, to achieve a balanced body slip, the non-steered rear wheels need to be utilized while retaining stability. The derivation leading up to (31) for the desired lumped slip angle $\alpha_{r}^{*}$ of the rear axle is new and relies on the reference variables $\theta_{v}^{*}$ and $\mu_{\min }$. The feedback control law (32) is applied to track $\alpha_{r}^{*}$, where the resulting desired yaw acceleration $\ddot{\psi}^{\text {des }}$ is handled by the Modified Hamiltonian Algorithm. Here, observations of optimal control of the 9DoF model in Fig. 4 are used to motivate the update law (37) that trades off achieving the desired yaw acceleration against increased center-of-mass acceleration in the direction $\theta_{v}^{*}$. Last in the Chassis-Level Control step, acceleration references for the individual wheels $\tilde{\theta}_{i}$ are computed in (39), being modified with respect to $\theta_{v}^{*}$ to take yaw-moment control into account.

Finally, the Wheel-Level Control (see Section V-B) allocates appropriate steering and braking signals based on the wheellevel acceleration references $\tilde{\theta}_{i}$ and the minimum friction coefficient $\mu_{\min }$. In this step, the new tire model (29) is used to determine desired front-wheel slip angles (43) from $\tilde{\theta}_{1}, \tilde{\theta}_{2}$, and $\mu_{\min }$, which in conjunction with a novel handling of nonequal front-wheel steering angles (41) is used to compute the steering-wheel rate. Finally, appropriate braking for each wheel is computed from $\tilde{\theta}_{i}$ and $\mu_{\mathrm{min}}$.

\section{A. Chassis-Level Control}

The task of the chassis-level controller is to maintain yaw stability and control the slip angles of the non-steered wheels, such that the force in the direction described by the acceleration reference $\theta_{v}^{*}$ can be maximized. The slip angles of the front wheels are controlled by steering, so the chassis-level controller only acts to control the slip angles of the rear wheels. The chassis-level control is closely based on the controller in [17], but uses the new tire model (29) to compute the desired slipangle reference. Another difference is that here the controller is only actively used to prevent rear slip angles larger than those desired, rather than also increasing the turn-in moment to achieve them. There are two motivations for this. Firstly, keeping the rear slip angles smaller increases the yaw stability of the vehicle. Secondly, the optimal behavior observed in [31] and here in Fig. 4 suggests that the optimal solution does not allocate significant tire forces to actively control the yaw moment.

1) Desired Yaw Acceleration: The tire forces are approximated as isotropic, i.e., $\mu_{x, i}=\mu_{y, i}$, which from (30) gives $\theta_{i}=\varphi_{i}$ (see Fig. 7). The slip angle for the rear axle that maximizes the force in the direction given by $\theta_{r}^{*}=\theta_{v}^{*}$ (see Fig. 9) is computed with (29) where $\alpha_{\mathrm{sl}}$ is given by the total sliding condition (20) using $\mu_{y, i}=\mu_{\min }$, i.e.,

$$
\alpha_{r}^{*}=\alpha_{\mathrm{sl}} \sin \left(\theta_{v}^{*}\right), \quad \alpha_{\mathrm{sl}}=\arctan \left(3 \mu_{\mathrm{min}} / \hat{C}_{\alpha}\right) .
$$

The error in the vehicle orientation $\psi$ is equal to the error in the body slip $\beta$ (see Fig. 9), where the body slip is defined as $\beta=\arctan \left(v_{y} / v_{x}\right)$. To ensure stability of the body slip, the 


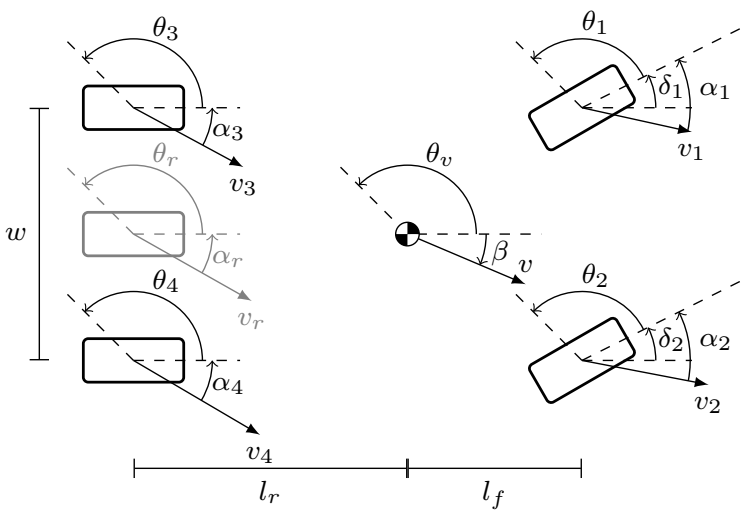

Fig. 9. Illustration of the vehicle with a lumped rear wheel in gray.

following control law for the desired yaw acceleration $\ddot{\psi}^{\text {des }}$ is obtained from the characteristic equation of a critically damped stable second-order system with poles at $-\omega$ :

$$
\ddot{\psi}^{\mathrm{des}}=-2 \omega \dot{\beta}^{e}-\omega^{2} \beta^{e} \text {. }
$$

Using the small-angle approximations $\alpha_{i} \approx-v_{y, i} / v_{x, i}$ and $\beta \approx v_{y} / v_{x}$, the body-slip error $\beta^{e}$ and its derivative are related to the desired lumped slip angle $\alpha_{r}^{*}$ of the rear axle by

$$
\begin{aligned}
& \beta^{e}=-\alpha_{r}^{*}+\frac{l_{r} \dot{\psi}-v_{y}}{v_{x}}, \\
& \dot{\beta}^{e}=-\dot{\alpha}_{r}^{*}+\frac{l_{r} \ddot{\psi}-a_{y}+v_{x} \dot{\psi}}{v_{x}}+\frac{\left(v_{y}-l_{r} \dot{\psi}\right)\left(a_{x}-v_{y} \dot{\psi}\right)}{v_{x}^{2}} .
\end{aligned}
$$

Given that the acceleration reference $\theta^{*}$ is constant in the global coordinates, the derivative of the desired lumped rear slip angle $\dot{\alpha}_{r}^{*}$ is derived from (31) to be

$$
\dot{\alpha}_{r}^{*}=\alpha_{\mathrm{sl}} \dot{\theta}_{v}^{*} \cos \left(\theta_{v}^{*}\right)=-\alpha_{\mathrm{sl}} \dot{\psi} \cos \left(\theta_{v}^{*}\right) .
$$

2) Control Allocation: To achieve the desired yaw acceleration $\ddot{\psi}$ des, the Modified Hamiltonian Algorithm (MHA) [13] is applied. Similar to (16), the optimal steering and braking input $u^{*}$ is the result of a weighted trade-off between maximizing the force in the direction described by the acceleration reference $\theta_{v}^{*}$, and controlling the yaw moment

$$
u^{*}=\underset{u \in U}{\arg \max }\left(\cos \left(\theta_{v}^{*}\right) F_{x}(u)+\sin \left(\theta_{v}^{*}\right) F_{y}(u)+\lambda M_{z}(u)\right) .
$$

Rather than finding $\lambda$ through optimization for an optimal trade-off as in [12], this parameter is as in [13], [17], [36] adapted online to effectively constrain the yaw moment $M_{z}$ to its desired value. Noting that the yaw moment $M_{z}$ is related to the yaw acceleration $\ddot{\psi}$ by $M_{z}=I_{z z} \ddot{\psi}$, where $I_{z z}$ is the yaw moment of inertia, $\lambda$ is adapted with the following two steps

$$
\begin{aligned}
& \lambda \rightarrow \lambda+S \tanh \left(\epsilon\left(\ddot{\psi}^{\text {des }}-\ddot{\psi}\right)\right), \\
& \lambda \rightarrow \begin{cases}0, & \text { if } \lambda \alpha_{r}^{*} \geq 0, \\
\lambda, & \text { otherwise },\end{cases}
\end{aligned}
$$

where the parameters $S$ and $\epsilon$ determine the change of $\lambda$ at each controller sampling instant. For the reasons discussed in

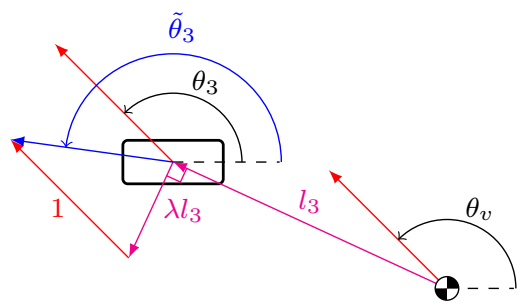

Fig. 10. Illustration of the computation of the acceleration reference $\tilde{\theta}_{3}$.

the beginning of Section V-A, the second update step prevents the controller from actively increasing the rear slip angle.

The yaw moment $M_{z}$ is linear in terms of the individual tire forces $\left(F_{x, i}, F_{y, i}\right)$

$$
M_{z}=\sum_{i=1}^{4}\left(l_{x, i} F_{y, i}-l_{y, i} F_{x, i}\right),
$$

where $\left(l_{x, i}, l_{y, i}\right)$ is the position of wheel $i$ relative to the center of mass. Assuming that the actuators controlling each wheel is independent of each other, problem (36) is, by replacing $F_{x}$, $F_{y}$, and $M_{z}$ with the contributions from individual tire forces, cascaded to the individual wheels

$$
\begin{aligned}
\tilde{\theta}_{i} & =\arctan 2\left(\sin \left(\theta_{v}^{*}\right)+\lambda l_{x, i}, \cos \left(\theta_{v}^{*}\right)-\lambda l_{y, i}\right)-\delta_{i}, \\
u_{i}^{*} & =\underset{u_{i} \in U_{i}}{\arg \max }\left(\cos \left(\tilde{\theta}_{i}\right) F_{x, i}\left(u_{i}\right)+\sin \left(\tilde{\theta}_{i}\right) F_{y, i}\left(u_{i}\right)\right),
\end{aligned}
$$

where the modified acceleration reference $\tilde{\theta}_{i}$ describes the direction in which to maximize the individual tire force to account for yaw moment (see an example for one of the wheels in Fig. 10) and is the output of the chassis-level controller (see Fig. 8). While the braking may be assumed to be independent between the tires insofar that the load transfer is affected, the common front steering input is handled in Section V-B1.

\section{B. Wheel-Level Control}

The front-axle steering and individual wheel braking inputs are obtained by solving (40) for the individual tires. The wheellevel control is split into computing the front-axle steering assuming optimal braking forces, and computing individual wheel braking given the nominal lateral tire forces.

1) Steering: In [16], a steering controller for at-the-limit maneuvers was developed that neglected yaw-moment control. This results in the ability to lump the front wheels together, since apart from slightly different slip angles because of the yaw rate of the vehicle and the track width, the steering signals for the two wheels end up close to identical. Based on [16], a controller for individual front-wheel steering and four-wheel braking was developed in [17]. The controller includes yawmoment control, resulting in significantly different desired front slip angles. If the front-wheel steering is independent, there is no problem in individually controlling each slip angle. To adapt the controller to a vehicle with equal front steering angles, the wheel that requests the least lateral force was given priority, with the motivation that the lower slip angle is required to free forces for braking. This adaptation was shown in [17] to result 
in similar slip angles as a method based on steering towards the local optimum.

In the high-fidelity CarMaker model used for simulation, the front-wheel steering angles are realistically not equal, but dependent on the steering geometry. The actual steering angles of the front tires are assumed to be measured, meaning an adaptation similar to that in [17] can be made. Here, the steering-rate request that reduces the sum of the front-wheel slip angles, $\alpha_{1}+\alpha_{2}$, is used. With the approximate steering ratio $k_{\delta}$ between the steering wheel and the front wheels, the selection of steering-wheel rate is made as

$$
\dot{\delta}_{\mathrm{sw}}= \begin{cases}k_{\delta} \dot{\delta}_{u, 1}, & \text { if } \dot{\delta}_{u, 1} \alpha_{\text {sign }}<\dot{\delta}_{u, 2} \alpha_{\text {sign }}, \\ k_{\delta} \dot{\delta}_{u, 2}, & \text { otherwise }\end{cases}
$$

where $\alpha_{\text {sign }}=\operatorname{sign}\left(\alpha_{1}+\alpha_{2}\right)$. To compute $\dot{\delta}_{u, i}$, a feedback control law is synthesized as in [37] with the feedback gain $K$ by the exponentially stable error dynamics

$$
\dot{\alpha}_{i}-\dot{\alpha}_{i}^{*}=-K\left(\alpha_{i}-\alpha_{i}^{*}\right),
$$

where the desired slip angle $\alpha_{i}^{*}$ is the slip angle that given optimal braking maximizes the force in the direction described by the acceleration reference $\tilde{\theta}_{i}$. The desired slip angle $\alpha_{i}^{*}$ for each front tire is computed similarly to the desired lumped rear slip angle in the chassis controller, using (29), (20), and (30) with $\mu_{y, i}=\mu_{\min }$ and the approximation $\mu_{x, i}=\mu_{y, i}$, i.e.

$$
\alpha_{i}^{*}=\alpha_{\mathrm{sl}} \sin \left(\tilde{\theta}_{i}\right), \quad \alpha_{\mathrm{sl}}=\arctan \left(3 \mu_{\mathrm{min}} / \hat{C}_{\alpha}\right) .
$$

By approximating $\dot{\tilde{\theta}}_{i} \approx \dot{\theta}_{i}=\dot{\theta}_{v}-\dot{\delta}_{i}$, the derivative of the desired slip angle of the steered front wheels is

$$
\dot{\alpha}_{i}^{*} \approx-\alpha_{\mathrm{sl}}\left(\dot{\psi}+\dot{\delta}_{i}\right) \cos \left(\tilde{\theta}_{i}\right) .
$$

With the lateral distance to the left and right wheelbase, $l_{y, i}=$ $\pm w / 2$, and making small-angle approximations, the desired steering-rate inputs given by (42) are

$$
\begin{aligned}
\dot{\delta}_{u, i} & =\frac{1}{1+\alpha_{\mathrm{sl}} \cos \left(\tilde{\theta}_{i}\right)}\left(K\left(\alpha_{i}^{*}-\alpha_{i}\right)-\alpha_{\mathrm{sl}} \dot{\psi} \cos \left(\tilde{\theta}_{i}\right)\right. \\
& \left.+\frac{a_{y}+v_{x} \dot{\psi}+l_{f} \ddot{\psi}}{v_{x}-l_{y, i} \dot{\psi}}+\frac{\left(v_{y}+l_{f} \dot{\psi}\right)\left(a_{x}-v_{y} \dot{\psi}-l_{y, i} \ddot{\psi}\right)}{\left(v_{x}-l_{y, i} \dot{\psi}\right)^{2}}\right) .
\end{aligned}
$$

2) Braking: The braking controller is equivalent to that in [16] but uses the tire model (19) to compute the nominal lateral tire force $F_{y 0, i}$ with $\mu_{y, i}=\mu_{\min }$. The friction-ellipse based model (22) is used to account for the trade-off between the braking force and the loss in lateral tire force. This model does not require the complex modeling of forces from combined longitudinal and lateral tire slips and yields an explicit analytical solution for the desired braking force. The braking force that maximizes the force in the direction given by the acceleration reference $\tilde{\theta}_{i}$, is found by describing the possible forces given by (22) for $\mu_{x, i}=\mu_{\min }$ with the angle $\varphi_{b, i}$,

$$
F_{x, i}=\mu_{\min } F_{z, i} \cos \left(\varphi_{b, i}\right), \quad F_{y, i}=F_{y 0, i} \sin \left(\varphi_{b, i}\right),
$$

where $\varphi_{b, i} \in[\pi / 2, \pi]$ if $F_{y 0, i}>0$, otherwise $\varphi_{b, i} \in[\pi, 3 \pi / 2]$. The optimal braking force $F_{x, i}^{*}$ is either found at one of the boundary values of $\varphi_{i}$, or if it is within the boundaries at [16]

$$
\varphi_{b, i}=\arctan 2\left(F_{y 0, i} \sin \left(\tilde{\theta}_{i}\right), \mu_{\min } F_{z, i} \cos \left(\tilde{\theta}_{i}\right)\right) .
$$



Fig. 11. Performance comparison between different models and controllers at different initial passing angles $\gamma$. Lower is better.

The braking torque $T_{i}$ applied to each wheel is computed by disregarding wheel dynamics such that

$$
T_{i}=F_{x, i}^{*} R_{e}
$$

\section{Vi. Simulation Results}

The controller developed in Section $\mathrm{V}$ is evaluated with simulation in CarMaker 7.1 .1 and is run at $1000 \mathrm{~Hz}$. The vehicle examined has parameters similar to a Ford Focus with the mass $1174 \mathrm{~kg}$ and other parameters listed in Table I. To allow for a reasonably large yaw acceleration while retaining stability of the body slip, the tuning parameter $\omega$ in (32) is set to 10 . The tuning parameters $S$ and $\epsilon$ in (37a) are set to 0.05 and 1 , respectively, for the yaw acceleration to converge to its desired value when the condition in (37b) is not active. The feedback gain $K$ in (45) is set to 19 . Increasing $K$ gives minor improvements, but should be balanced against noise and actuator limitations, as was done for a similar controller on a real vehicle [37], leading to the same gain $K$. For the case of straight-line braking, the real value of $\mu$ is used rather than $\mu_{\text {min }}$ to be close to optimal braking similar to an ABS system. While the yaw acceleration $\ddot{\psi}$ can be difficult to measure in practice, a rough estimate proves to be sufficient; it is approximated using the output from the chassis-level controller by

$$
\ddot{\psi}_{\mathrm{est}}=\frac{1}{I_{z z}} \sum_{i=1}^{4} \mu_{\mathrm{min}} F_{z, i}\left(l_{x, i} \sin \left(\tilde{\theta}_{i}\right)-l_{y, i} \cos \left(\tilde{\theta}_{i}\right)\right) .
$$

The compound tire-road friction coefficient $\mu$ of the vehicle is dependent on the vehicle state owing to the tire-friction dependence on tire load. To compare the performance obtained for the different models, the nominal longitudinal friction coefficient $\mu_{\text {nom }}=1.07$ is used. The road friction coefficient $\mu_{\text {road }}$ scales for different road conditions such that $\mu=\mu_{\text {road }} \mu_{\text {nom. }}$.

\section{A. Performance}

To evaluate the performance for varying passing angles $\gamma$, simulations are made using the same initial values as for the optimizations in Section III-B: the distance $A=20 \mathrm{~m}$ and the velocity $v=70 \mathrm{~km} / \mathrm{h}$. In Fig. 11, the minimum road-friction coefficient for which the controller is capable of avoiding the obstacle has been found and plotted in the style of Fig. 3. The developed controller in CarMaker (CM) performs close to that of numerical optimization of the 9DoF vehicle 
model, in particular for passing angles $\gamma$ below $10^{\circ}$. For the optimal particle used to determine when to switch strategy in the developed controller, straight-line braking outperforms the passing maneuver at $16.7^{\circ}$, while for the developed controller, it is found that straight-line braking outperforms the passing maneuver already for $\gamma>15.4^{\circ}$, causing a discontinuity in Fig. 11. Considering this difference, as well as the well known fact that the risk of severe injuries in a crash is lessened by reducing the velocity, the decision of when to perform which maneuver could take more variables into consideration.

Compared with the ideal passing maneuver for a particle where the vehicle dynamics has been neglected, the developed controller in CarMaker requires roughly $22 \%$ more friction to successfully pass the obstacle for $\gamma=5^{\circ}$, and $15 \%$ more for $\gamma=15^{\circ}$, with a close to linear relationship in between. An explanation for the relative decrease with increased passing angle is that a higher passing angle results in more focus on braking, with the braking forces of the vehicle being achieved relatively fast compared with the lateral tire forces.

\section{B. Behavior}

During the passing maneuver there are two indicator variables to monitor successful progress of obstacle avoidance. Referring to the algorithm overview in Fig. 8, in every time step it is checked whether the passing angle $\gamma \leq 0$, meaning that if the vehicle continues on a straight path it will not hit the obstacle. Further, the update of the controller variable $\mu_{\min }$ determines the minimum friction required for the rest of the maneuver. Thus, a successful avoidance maneuver is indicated by $\mu_{\min }$ approaching zero, since the danger of collision is then reducing, and at $\mu_{\min }=0$ the maneuver is complete. An example is seen in Fig. 12 where the blue curves show a maneuver that is handled in less than $0.8 \mathrm{~s}$ with $6 \mathrm{~m}$ remaining to the obstacle. Further, Fig. 12 shows that the path resulting from the developed controller, when operating at the limit friction (orange curve) for which avoidance is still feasible, is close to identical to that resulting from numerical optimization. Under nominal friction conditions (blue curve) the obstacle is cleared before reaching it, because the lateral tire force $F_{y, i}$ for a given slip angle $\alpha_{i}$ increases with increasing friction, so avoidance is achieved early when the friction is larger than the minimum friction coefficient $\mu_{\text {min }}$ used by the controller.

When the developed controller operates at the limit friction, the controller variable $\mu_{\text {min }}$ (dashed curves) in Fig. 12 is close to constant during the maneuver. The difference between the variable $\mu_{\mathrm{min}}$ in the limit case and the actual required friction coefficient $\mu$ (solid lines) is small, but the difference indicates that the controller cannot fully achieve the acceleration of the center of mass prescribed by $\mu$. One reason is that load transfer during cornering reduces the total cornering-force capability of the tires. The 9DoF model used in numerical optimization does not model this loss, and it can be seen that $\mu_{\text {min }}$ for the developed controller in the limit case is close to the friction obtained by numerical optimization. Seen by the initial increase in $\mu_{\mathrm{min}}$ for the limit-friction case, the particle model underestimates the required friction at the start of the maneuver, and thus the controller initially underutilizes the available forces compared to if $\mu$ was assumed to be known.
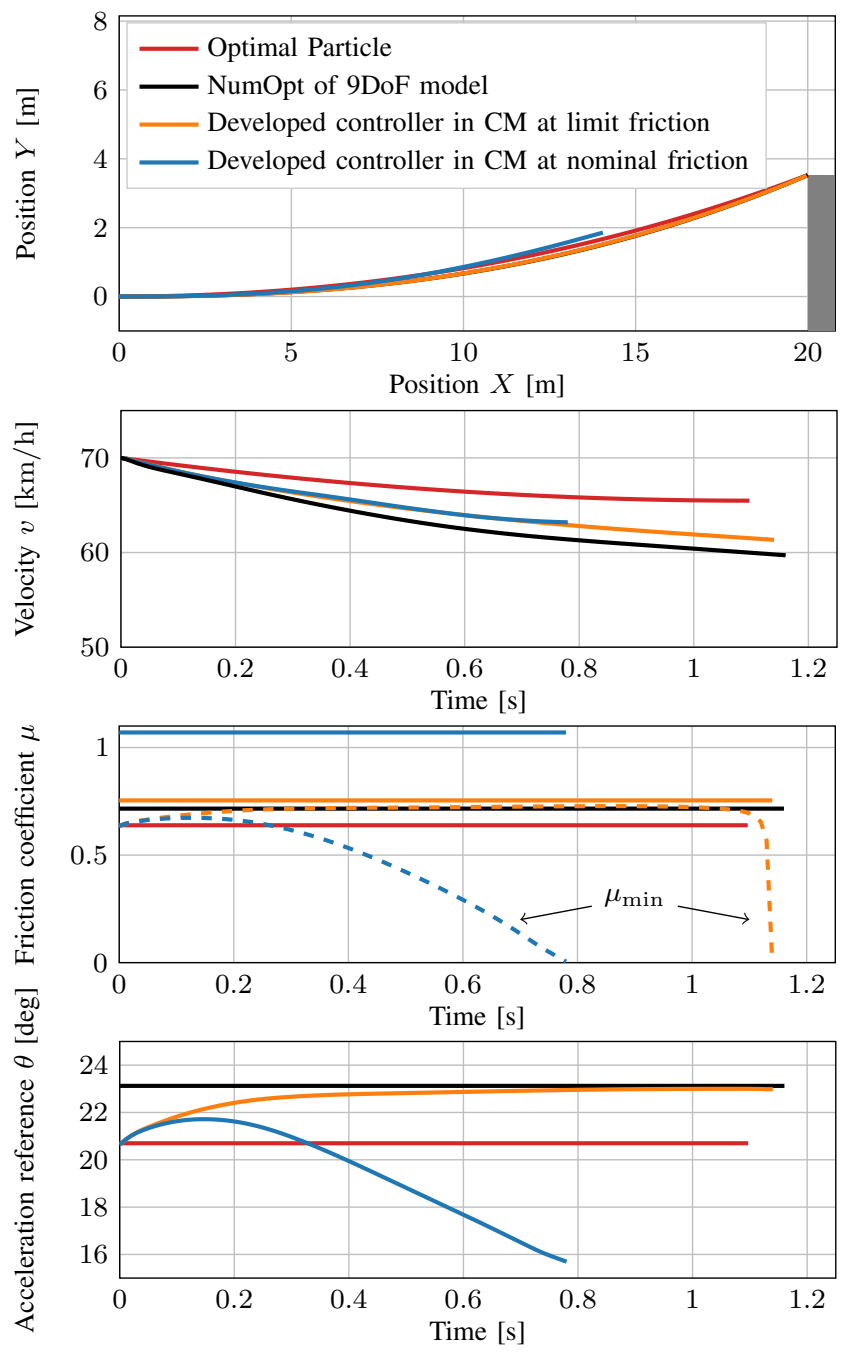

Fig. 12. Paths and high-level control variables during the avoidance maneuver with initial values $A=20 \mathrm{~m}, v=70 \mathrm{~km} / \mathrm{h}$, and $\gamma=10^{\circ}(B \approx 3.5 \mathrm{~m})$. The paths obtained for the numerical optimization and the developed controller at limit friction overlap. The variable $\mu_{\min }$ computed in the developed controller is shown as dashed curves.

The acceleration reference $\theta$ in the limit-friction case is in Fig. 12 seen to be close to that given by numerical optimization. While as observed in Fig. 5, the acceleration reference $\theta$ is slightly underestimated by the friction-limited particle model, it is corrected as the maneuver progresses. Fig. 13 shows that the body slip $\beta$ not exactly tracks its desired value as the tire forces are prioritized for collision avoidance, but the developed controller keeps $\beta$ small. The steering and braking commands from the wheel-level controller are shown in Fig. 14, where relatively heavier braking is observed on the right-hand side wheels in order to reduce the body slip.

The controller performs well, both at limit friction and at nominal friction, even when situation parameters are varied. One example is shown in Fig. 15 where the initial velocity is $120 \mathrm{~km} / \mathrm{h}$, i.e., significantly higher than the previous example of $70 \mathrm{~km} / \mathrm{h}$. Besides the demonstration of successful handling, it is interesting to see that the controller at this velocity puts more focus on the yaw control to maintain a low body slip (compare the yaw acceleration in Fig. 15 and Fig. 13). 

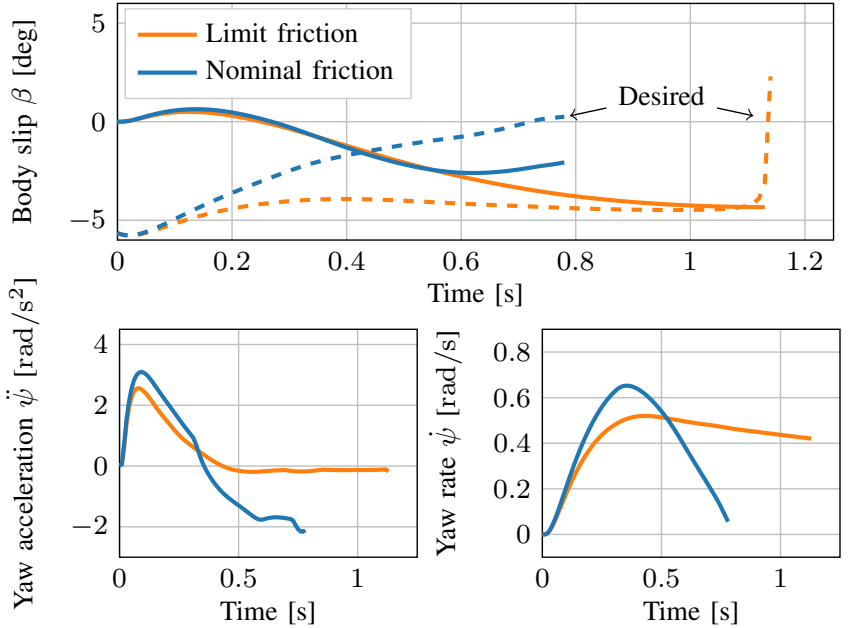

Fig. 13. Chassis-level control variables during the avoidance maneuver with initial values $A=20 \mathrm{~m}, v=70 \mathrm{~km} / \mathrm{h}$, and $\gamma=10^{\circ}(B \approx 3.5 \mathrm{~m})$. The desired body slip $\beta+\beta^{e}$ computed in the chassis-level controller is shown as dashed curves.


Fig. 14. Wheel-level control variables during the avoidance maneuver with initial values $A=20 \mathrm{~m}, v=70 \mathrm{~km} / \mathrm{h}$, and $\gamma=10^{\circ}(B \approx 3.5 \mathrm{~m})$.

\section{DISCUSSION \& CONCLUSIONS}

In the developed controller, the acceleration reference is retrieved from the optimal solution for single-obstacle avoidance of a friction-limited particle model, whose applicability is verified on a full vehicle model by using numerical optimization (see Section III). Since the solution for the optimal acceleration reference $\theta^{*}$ only requires knowing the passing angle $\gamma$, a sensor such as a mono-camera can easily determine it if it is assumed that the body slip is small. The minimum friction
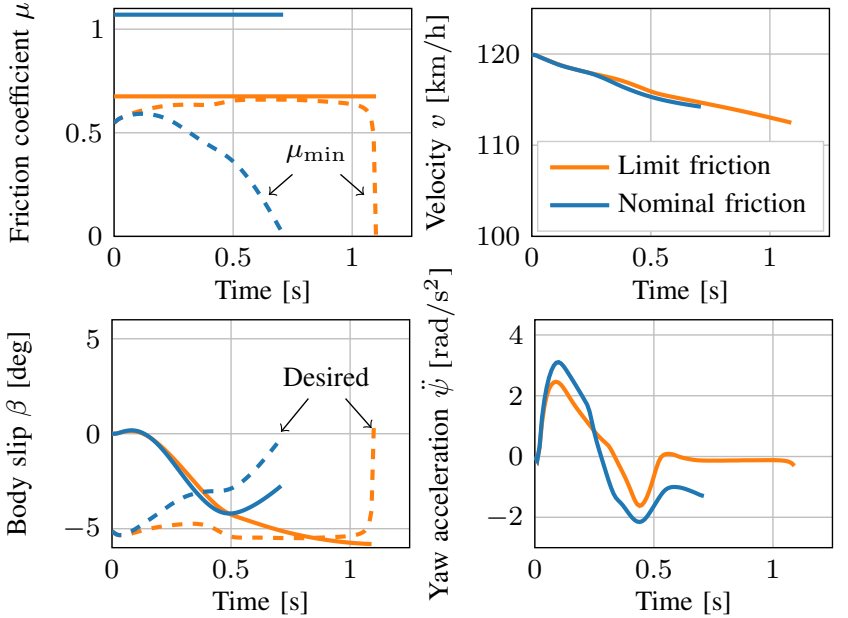

Fig. 15. The developed controller during the avoidance maneuver with initial values $A=35 \mathrm{~m}, v=120 \mathrm{~km} / \mathrm{h}$, and $\gamma=5^{\circ}(B \approx 3.1 \mathrm{~m})$. The variable $\mu_{\mathrm{min}}$ and the desired body slip $\beta+\beta^{e}$ computed in the developed controller are shown as dashed curves.

coefficient $\mu_{\min }$ required to follow the corresponding trajectory can be computed by additionally knowing the distance $d$ to the obstacle corner and the velocity $v$ of the vehicle.

The analytical friction-limit tire model derived in Section IV and used in the developed controller has the benefit of easily determining the desired slip angle. By the assumption that the tire force is at the friction limit, it is not necessary to numerically invert a full tire model, where there can be multiple combinations of longitudinal and lateral tire slips yielding the same forces. Further, the analytical friction-limit model shares its parameters with the pure-slip model (19). This is a distinct advantage over the empirical friction-limit model in [17], which requires a model fit at the friction limit.

Thanks to the nature of single-obstacle avoidance, the selection of models, approximations, and methods, the computation in the controller developed in Section V is fully explicit, making the computational time negligible. The wary approach allows the controller to perform successful collision avoidance without any estimation of the tire-road friction. Should the friction be high, then the controller makes use of this and clears the obstacle long before reaching it (as seen in the top plot in Fig. 12). This is an advantage of using an acceleration reference. Despite its modest computational time and independence of the friction, the developed controller is in Section VI shown to be able to handle situations close to the limit achieved by offline numerical optimization.

\section{REFERENCES}

[1] Y. Gao, T. Lin, F. Borrelli, E. Tseng, and D. Hrovat, "Predictive Control of Autonomous Ground Vehicles With Obstacle Avoidance on Slippery Roads," in ASME 2010 Dyn. Syst. and Control Conf. (DSCC), Cambridge, USA, 2010, pp. 265-272.

[2] J. Ji, A. Khajepour, W. W. Melek, and Y. Huang, "Path planning and tracking for vehicle collision avoidance based on model predictive control with multiconstraints," IEEE Trans. Veh. Technol., vol. 66, no. 2, pp. 952-964, 2017.

[3] X. He, Y. Liu, C. Lv, X. Ji, and Y. Liu, "Emergency steering control of autonomous vehicle for collision avoidance and stabilisation," Veh. Syst. Dyn., vol. 57, no. 8, pp. 1163-1187, 2019. 
[4] K. Berntorp, R. Quirynen, T. Uno, and S. D. Cairano, "Trajectory tracking for autonomous vehicles on varying road surfaces by friction-adaptive nonlinear model predictive control," Veh. Syst. Dyn., vol. 58, no. 5, pp. 705-725, 2020.

[5] Y. Hattori, E. Ono, and S. Hosoe, "Optimum vehicle trajectory control for obstacle avoidance problem," IEEE/ASME Trans. Mechatronics, vol. 11, no. 5, pp. 507-512, 2006

[6] S. C. Peters, "Optimal planning and control for hazard avoidance of front-wheel steered ground vehicles," Ph.D. dissertation, Dept. Mech. Eng., Massachusetts Inst. Technol., Cambridge, USA, 2012.

[7] F. Zeping and D. Jianmin, "Optimal lane change motion of intelligent vehicles based on extended adaptive pseudo-spectral method under uncertain vehicle mass," Adv. Mech. Eng., vol. 9, no. 7, pp. 1-15, 2017.

[8] J. Funke, M. Brown, S. M. Erlien, and J. C. Gerdes, "Collision avoidance and stabilization for autonomous vehicles in emergency scenarios," IEEE Trans. Control Syst. Technol., vol. 25, no. 4, pp. 1204-1216, 2017.

[9] L. Svensson, M. Bujarbaruah, N. R. Kapania, and M. Törngren, "Adaptive trajectory planning and optimization at limits of handling," in 2019 IEEE/RSJ Int. Conf. Intell. Robots and Syst. (IROS), 2019, pp. 3942 3948.

[10] M. Brown and J. C. Gerdes, "Coordinating tire forces to avoid obstacles using nonlinear model predictive control," IEEE Trans. Intell. Veh., vol. 5 , no. 1, pp. 21-31, 2020.

[11] K. Liu, J. Gong, A. Kurt, H. Chen, and U. Ozguner, "Dynamic modeling and control of high-speed automated vehicles for lane change maneuver," IEEE Trans. Intell. Veh., vol. 3, no. 3, pp. 329-339, 2018.

[12] D. Yang, B. Jacobson, M. Jonasson, and T. J. Gordon, "Closed-loop controller for post-impact vehicle dynamics using individual wheel braking and front axle steering," Int. J. Veh. Auton. Syst., vol. 12, no. 2 , pp. 158-179, 2014.

[13] Y. Gao, M. Lidberg, and T. J. Gordon, "Modified Hamiltonian algorithm for optimal lane change with application to collision avoidance." $M M$ Science J., no. MAR 2015, pp. 576-584, 2015.

[14] Y. Gao, T. Gordon, and M. Lidberg, "Optimal control of brakes and steering for autonomous collision avoidance using modified Hamiltonian algorithm," Veh. Syst. Dyn., vol. 57, no. 8, pp. 1224-1240, 2019.

[15] V. Fors, Y. Gao, B. Olofsson, T. J. Gordon, and L. Nielsen, "Real-time minimum-time lane change using the modified Hamiltonian algorithm,' in 26th Symp. Int. Assoc. Veh. Syst. Dyn. (IAVSD), Gothenburg, Sweden, 2019, pp. 1457-1465.

[16] V. Fors, B. Olofsson, and L. Nielsen, "Slip-angle feedback control for autonomous safety-critical maneuvers at-the-limit of friction," presented at 14th Int. Symp. Adv. Veh. Control (AVEC), Beijing, China, 2018.

[17] _ "Yaw-moment control at-the-limit of friction using individual frontwheel steering and four-wheel braking," in 9th IFAC Symp. Advances Automot. Control (AAC), vol. 52, no. 5, Orléans, France, 2019, pp. 458464.

[18] IPG Automotive GmbH, "Carmaker: Virtual testing of automobiles and light-duty vehicles," 2019. [Online]. Available: https://ipgautomotive.com/products-services/simulation-software/carmaker

[19] U. Kiencke and L. Nielsen, Automotive Control Systems, For Engine, Driveline, and Vehicle, 2nd ed. Berlin, Germany: Springer-Verlag, 2005.

[20] J. K. Subosits and J. C. Gerdes, "From the racetrack to the road: Realtime trajectory replanning for autonomous driving," IEEE Trans. Intell. Veh., vol. 4, no. 2, pp. 309-320, 2019.

[21] Z. Shiller and S. Sundar, "Emergency Lane-Change Maneuvers of Autonomous Vehicles," ASME. J. Dyn. Sys., Meas., Control, vol. 120, no. 1, pp. 37-44, 1998.

[22] Y. Hattori, E. Ono, and S. Hosoe, "An optimum vehicle trajectory control for obstacle avoidance with the shortest longitudinal traveling distance," in 2008 IEEE Int. Conf. Mech. and Automation (ICMA), Takamatsu, Japan, 2008, pp. 13-20.

[23] A. S. P. Singh and O. Nishihara, "Nondimensionalized univariate equation characterizing optimal state feedback control for collision avoidance," IEEE Trans. Intell. Transp. Syst., vol. 19, no. 10, pp. 3344-3359, 2018.

[24] K. Berntorp, B. Olofsson, K. Lundahl, and L. Nielsen, "Models and methodology for optimal trajectory generation in safety-critical roadvehicle manoeuvres," Veh. Syst. Dyn., vol. 52, no. 10, pp. 1304-1332, 2014.

[25] H. B. Pacejka, Tyre and Vehicle Dynamics, 2nd ed. Oxford, U.K.: Butterworth-Heinemann, 2006

[26] L. T. Biegler, A. M. Cervantes, and A. Wächter, "Advances in simultaneous strategies for dynamic process optimization," Chem. Eng. Science, vol. 57, no. 4, pp. 575-593, 2002.

[27] J. Åkesson, K.-E. Årzén, M. Gäfvert, T. Bergdahl, and H. Tummescheit, "Modeling and optimization with Optimica and JModelica.orgLanguages and tools for solving large-scale dynamic optimization problems," Comput. and Chem. Eng., vol. 34, no. 11, pp. 1737-1749, 2010.

[28] J. A. E. Andersson, J. Gillis, G. Horn, J. B. Rawlings, and M. Diehl, "CasADi - A software framework for nonlinear optimization and optimal control," Math. Program. Comput., vol. 11, no. 1, pp. 1-36, 2019.

[29] A. Wächter and L. T. Biegler, "On the implementation of an interior-point filter line-search algorithm for large-scale nonlinear programming," Math. Program., vol. 106, no. 1, pp. 25-57, 2006.

[30] "HSL. A collection of Fortran codes for large scale scientific computation," 2017, Accessed: 2017-02-15. [Online]. Available: http://www.hsl.rl.ac.uk/

[31] V. Fors, B. Olofsson, and L. Nielsen, "Attainable force volumes of optimal autonomous at-the-limit vehicle manoeuvres," Veh. Syst. Dyn., vol. 58, no. 7, pp. 1101-1122, 2020.

[32] M. Jonasson, J. Andreasson, B. Jacobson, and A. Stensson Trigell, "Global force potential of over-actuated vehicles," Veh. Syst. Dyn., vol. 48, no. 9, pp. 983-998, 2010.

[33] J. Y. Wong, Theory of Ground Vehicles, 4th ed. New York, NY, USA: Wiley, 2008

[34] E. Bakker, L. Nyborg, and H. B. Pacejka, "Tyre modelling for use in vehicle dynamics studies," in SAE Int. Congr. and Expo., Detroit, USA, 1987.

[35] J. Svendenius, "Tire modeling and friction estimation," Ph.D. dissertation, Dept. Autom. Control, Lund University, Lund, Sweden, 2007, No. TFRT1077.

[36] A. Arikere, D. Yang, and M. Klomp, "Optimal motion control for collision avoidance at left turn across path/opposite direction intersection scenarios using electric propulsion,” Veh. Syst. Dyn., vol. 57, no. 5, pp. 637-664 2019.

[37] J. K. Subosits and J. C. Gerdes, "A synthetic input approach to slip angle based steering control for autonomous vehicles," in 2017 Amer. Control Conf. (ACC), Seattle, USA, 2017, pp. 2297-2302.



Victor Fors received the M.Sc. degree in Engineering Physics in 2015 from Lund University, Sweden. Since 2016 he is a Ph.D. student at the Division of Vehicular Systems, Linköping University. His main research interests are in vehicle dynamics, control, active safety, and autonomy.

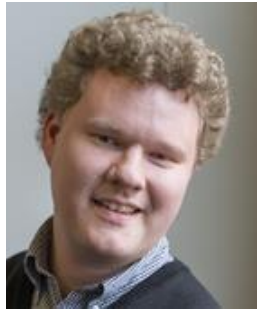

Björn Olofsson received the M.Sc. degree in Engineering Physics in 2010 and the Ph.D. degree in Automatic Control in 2015, both from Lund University, Sweden. He is currently a researcher at the Division of Vehicular Systems, Linköping University and at the Department of Automatic Control, Lund University. His research interests are in motion control for robots and vehicles, optimal control, system identification, and statistical sensor fusion.

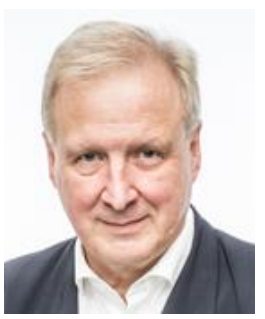

Lars Nielsen received his M.Sc. in engineering physics in 1979 and his Ph.D. degree in automatic control in 1985, both from Lund University. Since 1992 he is professor of Vehicular Systems holding the Sten Gustafsson chair at Linköping University. His main research interests are in automotive modeling, control, safety maneuvers, and autonomy. 